\title{
Role of Visual Field Testing and OCT in Evaluation of Brimonidine as a Neuroprotector in Primary Open Angle Glaucoma
}

\author{
Moustafa Kamal Nassar, Hany Ahmed Khairy, Sameh Mohamed El Gohary, Ahmed Mohamed Fawzy Deif * \\ Department of Ophthalmology, Faculty of Medicine, Menoufia University, Menoufia, Egypt \\ *Corresponding author: Ahmed Mohamed Fawzy Deif, Mobile: (+20)1227413780, Email: ahmedfawzyeyedr@gmail.com
}

\begin{abstract}
Background: Glaucoma is the leading cause of global irreversible blindness optic neuropathy and retinal ganglion cell (RGC) death are the hallmarks of glaucoma. If intraocular pressure (IOP) is held within "normal" ranges, the disease process and RGC death can still progress in some patients. Neuroprotective therapies for glaucoma should aim to halt RGC loss thus preventing further structural and functional damage of the optic nerve

Objective: The aim of the current work was to study the ability of visual field testing and optical coherence tomography to reveal the neuroprotective role of brimonidine.

Patients and Methods: This prospective randomized controlled trial study included a total of 98 eyes from 50 patients affected by primary open-angle glaucoma (POAG). They were recruited from a larger population of 70 POAG patients, attending at Menofiya University Hospitals.

Results: The use of visual field-testing parameters have revealed that brimonidine had insignificantly halted glaucoma progression: MD (-0.1115 dB and -.2465 dB), VFI (-0.5882 and-1.1471) and PSD (0.1729 and 0.1894). On the other hand, some OCT parameters: average RNFL thickness (-0.7273 u and-2.2121 u), inferior RNFL thickness $(-0.735 \mathrm{u}$ and $-1.559 \mathrm{u})$, superior RNFL thickness $(-0.6364 \mathrm{u}$ and $-1.4848 \mathrm{u})$, rim area $\left(-0.0162 \mathrm{~mm}^{2}\right.$ and $\left.-0.0797 \mathrm{~mm}^{2}\right)$, average cup disc ratio (-0.01765 and 0.01824) and vertical cup disc ratio (-0.0167 and 0.0321$)$ for study and control groups respectively, gave promising neuroprotective results for brimonidine.

Conclusion: It could be concluded that the use of visual field-testing parameters (MD, VFI and PSD) has revealed that brimonidine had insignificantly halted glaucoma progression.
\end{abstract}

Keywords: Brimonidine, Primary open angle glaucoma, Neuroprotection

\section{INTRODUCTION}

Glaucoma is the leading cause of global irreversible blindness. Over 79 million people worldwide are expected to be affected by primary open angle glaucoma in 2020 and 111.8 million by $2040^{(\mathbf{1})}$.

Optic neuropathy and RGC death are the hallmarks of glaucoma ${ }^{(2)}$. Elevated intraocular pressure has remained a clinical focus in glaucoma. However, the relationship between IOP reduction and glaucoma damage is less than clear. If IOP is held within "normal" ranges, the disease process and RGC death can still progress in some patients ${ }^{(3)}$.

Neuroprotective therapies for glaucoma should aim to halt RGC loss thus preventing further structural and functional damage of the optic nerve ${ }^{(4)}$. Since its introduction in 1996, the use of brimonidine has become an increasingly popular ocular hypotensive therapy ${ }^{(5)}$. Preclinical studies have clarified numerous mechanisms for neuroprotection that brimonidine may exhibit. Moreover, many clinical trials on brimonidine as a neuroprotection agent have been conducted either within or outside the field of glaucoma.

The aim of this study was to investigate the role of visual field testing and OCT in evaluation of brimonidine eye drops as a neuroprotector in primary open-angle glaucoma (POAG) by providing functional and structural evidence of brimonidine ability to slow the progression of POAG throughout a non-IOP related mechanism.

\section{PATIENTS AND METHODS}

This prospective randomized controlled trial study included a total of 98 eyes from 50 patients affected by POAG, attending at Menofiya University Hospitals.

\section{Ethical Consideration:}

This study was ethically approved by the Menofiya University Ethics Committee. Written informed consent of all the subjects was obtained. This work has been carried out in accordance with The Code of Ethics of the World Medical Association (Declaration of Helsinki) for studies involving humans.

\section{Inclusion Criteria:}

The study included 98 eyes from 50 patients affected by POAG were recruited from a larger population of 70 POAG patients based on the following inclusion criteria:

- Best-corrected visual acuity of $\geq 20 / 40$ (Refractive errors below $+/-6$ diopters).

- No history or evidence of retinal or nonglaucomatous optic nerve diseases, laser therapy, or ocular surgery.

- Absence of any contraindication for the medications used in this study.

- Each patient had a definite diagnosis of glaucoma based on glaucomatous damage to the optic disc 
(defined as neuroretinal rim thinning, notching, excavation, or RNFL defect) and abnormal VF. At least two VF tests (Humphrey; Carl Zeiss Meditec, Inc.) using the SITA standard 24-2 program were required. VFs were abnormal at the time of diagnosis if any of the following was true: glaucoma hemifield test result reliably outside normal limits; pattern standard deviation (PSD) $\mathrm{P}$ $<5 \%$; cluster of three or more non-edge points in the PSD plot in the superior or inferior hemifield with $\mathrm{P}<5 \%$, including one or more with $\mathrm{P}<1 \%$.

- Gonioscopy revealed open angle and Pretreatment IOP $22 \mathrm{mmHg}$ or more.

- If both eyes of the same patient accomplished the criteria, both were included and analyzed independently.

Exclusion Criteria: included loss of any of inclusion criteria and failure of compliance to treatment plan or follow up schedule.

All included subjects underwent a medical and ocular history and an ophthalmic examination, including refraction, measurement of best-corrected visual acuity testing, external ocular examination, slitlamp biomicroscopy (Topcon Medical Systems, Japan), IOP measurement (Goldmann applanation tonometer, Haag-Streit diagnostics, Switzerland), gonioscopy (Three Mirror Universal Lens, Ocular Instruments Inc., USA), fundus examination ( Volk 90 D non-contact Slit Lamp lens, Volk Optical, Inc., USA ), visual field testing (HFA II, Carl Zeiss Meditec Inc., USA), and spectral domain optical coherence tomography (CIRRUS, Carl Zeiss Meditec Inc., USA).

The included subjects were randomly divided into two groups; Study Group: consisted of 30 patients (59 eyes) who were treated by a fixed combination of brimonidine tartarate / timolol maleate ophthalmic solution $0.2 \% / 0.5 \%$ and Control Group: consisted of 20 patients (39 eyes) were treated by a fixed combination of dorzolamide hydrochloride / timolol maleate ophthalmic solution $2 \% / 0.5 \%$.

Follow up visits were spaced at one, three- and six-months intervals.

Each follow up visit included IOP measurement, visual field testing, and spectral domain optical coherence tomography examination for $\mathrm{ONH}$, RNFL and Ganglion cell analysis.

During follow up period, 3 patients (6 eyes) from the study group and 3 patients (6 eyes) from the control group have dropped off the follow up visits and were excluded from results.

Standardized visual field testing was performed with static automated white-on-white threshold perimetry (SITA Standard 24-2, Humphrey Field Analyzer II; Carl Zeiss Meditec). A visual field was defined as reliable when fixation losses were less than $20 \%$, and false-positive and false-negative rates were less than $33 \%$.
Using Cirrus HD-OCT (Carl Zeiss Meditec, Dublin, CA, USA), only good-quality scans with wellfocused images, no overt misalignment and no overt decentration of the measurement circle location around the optic disc have been accepted.

-An optic disc cube was obtained from a threedimensional data set composed of 200 A scans from each of $200 \mathrm{~B}$ scans covering a 6-mm2 area centered on the optic disc. The software automatically determines the center of the disc and then positions a 3.46-mm diameter calculation circle for RNFL thickness measurement. Using these data, SD-OCT provided thickness parameters including average, 12 clock-hour sectors, and four quadrant sectors. The software the analyzes the values, compares them to the internal normative database, and generates a thickness map, deviation map, and significance map that are color-coded to match RNFL thickness, with values within the normal range in green $(\mathrm{P}=5 \%-95 \%)$, borderline values in yellow $(\mathrm{P}=1 \%-5 \%)$, and values outside the normal range in red $(\mathrm{P}<1 \%)$.

An internal analysis algorithm measures $\mathrm{ONH}$ parameters, such as the rim area, the cup-to disc area ratio, and the vertical cup-to-disc diameter ratio and cup volume.

The Cirrus HD-OCT (software v. 6.0.0; Carl Zeiss Meditec Inc., Dublin, California, USA) segmentation software calculates the thickness of the macular ganglion cell-inner plexiform layer from an elliptical annulus centered on the fovea (thickness map) and calculates the thicknesses of the combined ganglion cell and IPL using the Macular Cube $512 \times$ 128 scan patterns.

Based on 3-dimensional data generated from the macular cube scan protocol, the algorithm identifies the outer boundary of RNFL and the outer boundary of inner plexiform layer at the macular region; this segmented layer yielded macular GCIPL thickness. The scan generates data in a $6 \mathrm{~mm} \times 6 \mathrm{~mm}$ grid. The results are compared to normative data (Deviation map). The ganglion cell analysis segmentation algorithm divides the elliptical annulus of the Thickness Map into 6 equal sectors expressed in micrometers.

\section{Statistical analysis of the data}

Data were collected about changes occurring on the following parameters: IOP. Visual field testing: Mean Deviation, Visual Field Index and Pattern Standard Deviation. OCT: average RNFL thickness, inferior RNFL thickness, superior RNFL thickness, rim area, average cup disc ratio, vertical cup disc ratio cup volume, average GCL+ IPL thickness and minimum GCL+ IPL thickness.

Changes were classified into: First change: is the difference between the values of any given parameter at the baseline visit and the first follow up visit at the first month. Second change: is the difference between the values of any given parameter at the first follow up 
visit and the second follow up visit at the third month. Third change: is the difference between the values of any given parameter at the second follow up visit and the third follow up visit at the sixth month. Absolute change: which is the sum of first, second and third changes.

Data were fed to the computer using IBM SPSS software package version 23.0.

\section{RESULTS}

mean age in study group was 54.15 years, while the mean age in control group was 52.33 years, with no statistically significant difference between the two groups ( $\mathrm{p}=0.838$ ). However, female predominance was noted in both groups, more evident in the study group $(73.6 \%)$ than in the control group (60.6\%).

\section{Intraocular pressure:}

There was no statistically significant difference $(p$-value $=0.374)$ between the study group and the control group regarding IOP at the initial visit (24.79 $\mathrm{mm} \mathrm{Hg}$ and $25.06 \mathrm{~mm} \mathrm{Hg}$ respectively). Moreover, the absolute decrease in IOP at the end of the study was in favor for the control group in comparison to the study group (-7.52 $\mathrm{mm} \mathrm{Hg}$ and $-7.33 \mathrm{~mm} \mathrm{Hg}$ respectively), however this difference was statistically insignificant (p-value $=0.404)$.

\section{Visual field testing parameters:}

There was no statistically significant difference ( $\mathrm{p}$ value $=0.276$ ) between the study group and the control group regarding Mean Deviation at the initial visit ($4.0624 \mathrm{~dB}$ and $-4.7791 \mathrm{~dB}$ respectively).

Changes in absolute mean values throughout the study showed a lesser absolute decrease in mean MD $(-0.1115 \mathrm{~dB}$ and $-.2465 \mathrm{~B})$, a lesser absolute decrease in mean VFI (-0.5882 and-1.1471) and a lesser absolute increase in mean PSD (0.1729 and 0.1894) within the study group compared to that in the control group respectively. However, these changes were found to be statistically insignificant with $\mathrm{p}$-values of $0.647,0.258$ and 0.919 respectively. 
Table (1): Changes in VF parameters throughout the study and their statistical significance.

\begin{tabular}{|c|c|c|c|c|c|c|c|c|c|c|c|c|c|}
\hline & & $\begin{array}{c}\text { MD } \\
\text { Mean }\end{array}$ & $\begin{array}{l}\text { MD } \\
\text { SD }\end{array}$ & $\begin{array}{c}\text { MD } \\
\mathbf{t} \\
\end{array}$ & $\begin{array}{c}\text { MD } \\
\text { Sig. (2- } \\
\text { tailed) }\end{array}$ & $\begin{array}{c}\text { VFI } \\
\text { Mean }\end{array}$ & $\begin{array}{l}\text { VFI } \\
\text { SD }\end{array}$ & $\begin{array}{c}\text { VFI } \\
\mathbf{t}\end{array}$ & $\begin{array}{c}\text { VFI } \\
\text { Sig. (2- } \\
\text { tailed) }\end{array}$ & $\begin{array}{c}\text { PSD } \\
\text { Mean }\end{array}$ & $\begin{array}{c}\text { PSD } \\
\text { SD }\end{array}$ & $\begin{array}{c}\text { PSD } \\
\mathbf{t}\end{array}$ & $\begin{array}{l}\text { PSD } \\
\text { Sig. (2- } \\
\text { tailed) }\end{array}$ \\
\hline Pair 1 & 1st_change_test & .1603 & 1.55855 & .664 & .511 & .1176 & 1.85480 & 1.044 & .304 & .0300 & .68883 & -1.280 & .209 \\
\hline \multirow{2}{*}{ Pair 2} & 2nd_change_test & -.2924 & 1.52690 & \multirow{2}{*}{$-.856-$} & \multirow{2}{*}{.398} & -.1176 & 1.71914 & \multirow{2}{*}{.651} & \multirow{2}{*}{.520} & -.0315 & .65486 & \multirow{2}{*}{.955} & \multirow[b]{2}{*}{.346} \\
\hline & 2nd_change_control & -.1029 & 1.08271 & & & -.4118 & 1.98659 & & & -.1656 & .65486 & & \\
\hline \multirow{2}{*}{ Pair 3} & 3rd_change_test & .0206 & .55209 & \multirow{2}{*}{.684} & \multirow{2}{*}{.499} & -.5882 & 1.30541 & \multirow{2}{*}{$-1.158-$} & \multirow{2}{*}{.255} & .1744 & .56516 & \multirow{2}{*}{.519} & \multirow{2}{*}{.607} \\
\hline & 3rd_change_control & -.0932 & .81294 & & & -.3235 & 1.17346 & & & .1185 & .33146 & & \\
\hline Pair 4 & Absolute change_control & -.2465 & 1.32863 & .463 & .647 & 1.1471 & 1.94051 & 1.151 & .258 & .1894 & .56032 & -.103 & .919 \\
\hline
\end{tabular}

\section{OCT parameters:}

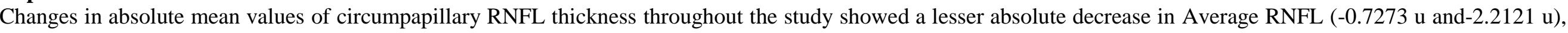

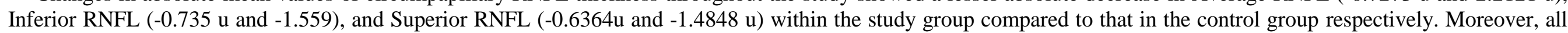
these changes were found to be statistically significant with p-values of $0.015,0.016$ and 0.042 respectively.

Table (2): Changes in RNFL parameters throughout the study and their statistical significance.

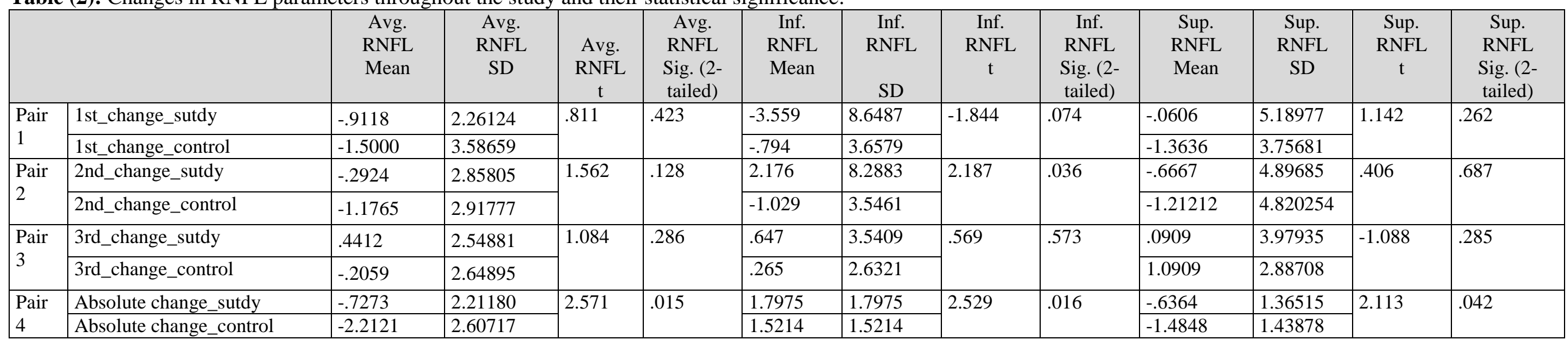

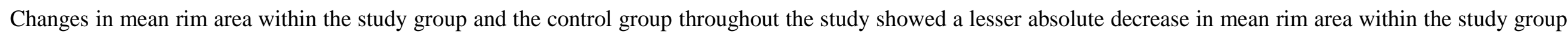
compared to that in the control group $(-0.0162 \mathrm{~mm} 2$ and- $-0.0797 \mathrm{~mm} 2$ respectively $)$. These changes were found to be statistically significant with $\mathrm{p}$-value $=0.025$.

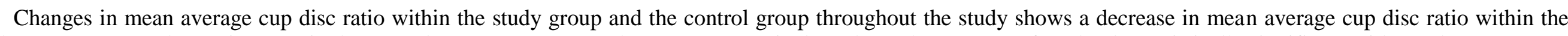

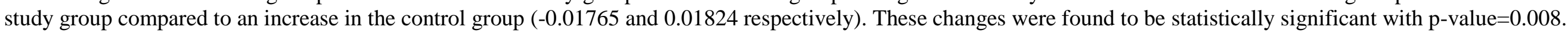

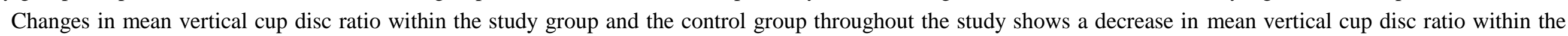

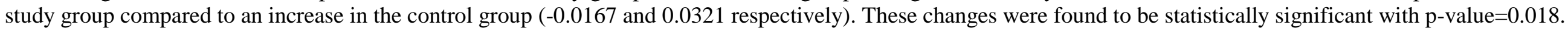


Changes in mean cup volume within the study group and the control group throughout the study shows a lesser increase in mean cup volume within the study group compared to that in the control group $(0.00312 \mathrm{~mm} 3$ and $0.00759 \mathrm{~mm} 3$ respectively $)$. These changes were found to be statistically insignificant with p-value $=0.784$.

Table (3): Changes in ONH parameters throughout the study and their statistical significance.

\begin{tabular}{|c|c|c|c|c|c|c|c|c|c|c|c|c|c|c|c|c|c|}
\hline & & $\begin{array}{c}\text { Rim } \\
\text { Area } \\
\text { Mean }\end{array}$ & $\begin{array}{c}\text { Rim } \\
\text { Area } \\
\text { SD }\end{array}$ & $\begin{array}{c}\text { Rim } \\
\text { Area } \\
\mathbf{t} \\
\end{array}$ & $\begin{array}{c}\text { Rim } \\
\text { Area Sig. } \\
\text { (2-tailed) }\end{array}$ & $\begin{array}{l}\text { Avg. } \\
\text { CD } \\
\text { Mean }\end{array}$ & $\begin{array}{l}\text { Avg. } \\
\text { CD } \\
\text { SD }\end{array}$ & $\begin{array}{c}\text { Avg. } \\
\text { CD } \\
\text { t }\end{array}$ & $\begin{array}{c}\text { Avg. } \\
\text { CD Sig. } \\
(2- \\
\text { tailed) }\end{array}$ & $\begin{array}{l}\text { VCD } \\
\text { Mean }\end{array}$ & $\begin{array}{c}\text { VCD } \\
\text { SD }\end{array}$ & $\begin{array}{c}\text { VCD } \\
\mathbf{t}\end{array}$ & $\begin{array}{c}\text { VCD } \\
\text { Sig. (2- } \\
\text { tailed) }\end{array}$ & $\begin{array}{l}\text { Cup } \\
\text { VOL } \\
\text { Mean }\end{array}$ & $\begin{array}{c}\text { Cup } \\
\text { VOL } \\
\text { SD }\end{array}$ & $\begin{array}{c}\text { Cup } \\
\text { VOL } \\
\mathbf{t}\end{array}$ & $\begin{array}{c}\text { Cup } \\
\text { VOL } \\
\text { Sig. (2- } \\
\text { tailed) }\end{array}$ \\
\hline \multirow{2}{*}{$\begin{array}{l}\text { Pair } \\
1\end{array}$} & 1st_change_sutdy & .0047 & .08436 & \multirow{2}{*}{1.068} & \multirow{2}{*}{.293} & -.01672 & .036137 & \multirow{2}{*}{-.069} & \multirow{2}{*}{.945} & .01987 & .037394 & \multirow{2}{*}{.992} & \multirow{2}{*}{.329} & -.00335 & .035418 & \multirow{2}{*}{.600} & \multirow{2}{*}{.552} \\
\hline & 1st_change_control & -.0262 & .14185 & & & \begin{tabular}{|l|}
-.00747 \\
\end{tabular} & .048273 & & & .0073 & .06583 & & & \begin{tabular}{|l|}
-.00891 \\
\end{tabular} & .054744 & & \\
\hline \multirow{2}{*}{$\begin{array}{l}\text { Pair } \\
2\end{array}$} & 2nd_change_sutdy & -.0106 & .08920 & \multirow{2}{*}{.657} & \multirow{2}{*}{.516} & .015176 & .090489 & \multirow{2}{*}{.895} & \multirow{2}{*}{.377} & -.018255 & .044251 & \multirow{2}{*}{$-.923-$} & \multirow{2}{*}{.363} & .00415 & .063167 & \multirow{2}{*}{-.736} & \multirow{2}{*}{.467} \\
\hline & 2nd_change_control & -.0232 & .07571 & & & .01335 & .023338 & & & .0127 & .04148 & & & .01426 & .042795 & & \\
\hline \multirow{2}{*}{$\begin{array}{l}\text { Pair } \\
3\end{array}$} & 3rd_change_sutdy & -.0103 & .06520 & \multirow{2}{*}{1.470} & \multirow{2}{*}{.151} & -.01611 & .090583 & \multirow{2}{*}{$\begin{array}{c}- \\
1.098\end{array}$} & \multirow{2}{*}{.280} & -.01636 & .091959 & \multirow{2}{*}{$-1.071-$} & \multirow{2}{*}{.292} & .00232 & .033591 & \multirow{2}{*}{.011} & \multirow{2}{*}{.991} \\
\hline & 3rd_change_control & -.0303 & .05036 & & & .01235 & .042857 & & & .0121 & .04285 & & & .00224 & .029921 & & \\
\hline \multirow{2}{*}{$\begin{array}{l}\text { Pair } \\
4\end{array}$} & \begin{tabular}{|l|}
$\begin{array}{l}\text { Absolute } \\
\text { change_sutdy }\end{array}$ \\
\end{tabular} & -.0162 & .07909 & \multirow{2}{*}{2.348} & \multirow{2}{*}{.025} & -.01765 & .073405 & \multirow{2}{*}{2.831} & \multirow{2}{*}{.008} & -.0167 & .08859 & \multirow{2}{*}{$-2.488-$} & \multirow{2}{*}{.018} & .00312 & .061434 & \multirow{2}{*}{-.277} & \multirow{2}{*}{.784} \\
\hline & $\begin{array}{l}\begin{array}{l}\text { Absolute } \\
\text { change_control }\end{array} \\
\end{array}$ & -.0797 & .15083 & & & .01824 & .045625 & & & .0321 & .09048 & & & .00759 & .066412 & & \\
\hline
\end{tabular}

Changes in absolute mean values of GCIPL thickness throughout the study showed a lesser absolute decrease in Average GCIPL thickness (-0.9412 and -1.7059 $\mathrm{u}$ ) and Minimum GCLIPL thickness (-0.7801u and $-1.2701 \mathrm{u})$ within the study group compared to that in the control group respectively. However, these changes were found to be statistically insignificant with p-values of 0.264 and 0.245 respectively.

Table (4):Changes in GCIPL parameters throughout the study and their statistical significance.

\begin{tabular}{|c|c|c|c|c|c|c|c|c|c|}
\hline 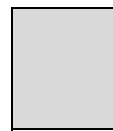 & & $\begin{array}{l}\text { Avg. } \\
\text { RGCIPL } \\
\text { Mean }\end{array}$ & $\begin{array}{l}\text { Avg. } \\
\text { RGCIPL } \\
\text { SD } \\
\end{array}$ & $\begin{array}{c}\text { Avg. } \\
\text { RGCIPL } \\
t \\
\end{array}$ & $\begin{array}{c}\text { Avg. } \\
\text { RGCIPL } \\
\text { Sig. (2-tailed) }\end{array}$ & $\begin{array}{c}\text { Min. } \\
\text { RGCIPL } \\
\text { Mean } \\
\end{array}$ & $\begin{array}{c}\text { Min. } \\
\text { RGCIPL } \\
\text { SD } \\
\end{array}$ & $\begin{array}{c}\text { Min. } \\
\text { RGCIPL } \\
\mathbf{t} \\
\end{array}$ & $\begin{array}{c}\text { Min. } \\
\text { RGCIPL } \\
\text { Sig. (2-tailed) }\end{array}$ \\
\hline \multirow{2}{*}{ Pair 1} & 1st_change_sutdy & -.3529 & 2.96323 & \multirow{2}{*}{.749} & \multirow{2}{*}{.458} & -.9521 & 1.717 & \multirow{2}{*}{.758} & \multirow{2}{*}{.494} \\
\hline & 1st_change_control & -2.6765 & 6.67751 & & & -1.2444 & 1.908 & & \\
\hline \multirow{2}{*}{ Pair 2} & 2nd_change_sutdy & -.6176 & 2.20213 & \multirow{2}{*}{$-.890-$} & \multirow{2}{*}{.379} & -.1728 & 2.418 & \multirow{2}{*}{-.833} & \multirow{2}{*}{.390} \\
\hline & 2nd_change_control & 1.4118 & 6.36333 & & & .2459 & 2.245 & & \\
\hline \multirow{2}{*}{ Pair 3} & 3rd_change_sutdy & .0294 & 1.50726 & \multirow{2}{*}{1.538} & \multirow{2}{*}{.132} & .3448 & 2.186 & \multirow{2}{*}{1.713} & \multirow{2}{*}{.127} \\
\hline & 3rd_change_control & -.4412 & 1.86176 & & & -.2716 & 1.975 & & \\
\hline Pair 4 & Absolute change_control & -1.7059 & 2.55283 & 1.133 & .264 & -1.2701 & 2.270 & 1.151 & .245 \\
\hline
\end{tabular}




\section{DISCUSSION}

Neuroprotection studies in glaucoma were challenged to provide clear structural and functional endpoints amenable to elaborate results ${ }^{(6)}$. However, the outcomes of the completed clinical trials were not completely satisfactory ${ }^{(7)}$.

Clinical detection of neuroprotection requires optimized measurement of both structural and functional endpoints to reveal differential rates of neurodegeneration in treated and control groups ${ }^{(8)}$. The relationship between structure and function in glaucoma is complex .This is due to the different scales of measurement for structure and function ${ }^{(9)}$.

In this study, the use of visual field testing parameters (MD, VFI and PSD) has revealed that brimonidine had insignificantly halted glaucoma progression. On the other hand, some OCT parameters (average RNFL thickness, inferior RNFL thickness, superior RNFL thickness, rim area, average cup disc ratio and vertical cup disc ratio) gave promising neuroprotective results for brimonidine, while other OCT parameters ( cup volume, average GCIPL thickness and minimum GCIPL thickness) gave statistically insignificant neuroprotective results for brimonidine..

In this study, within group and in-between group results of SAP (central 24-2 SITA standard) and SDOCT (RNFL, ONH and RGCIPL) values were studied over 6 months.

To reveal the neuroprotective role of brimonidine, Tsai and Chang needed 1 year to using only structural endpoints (RNFL thickness) ${ }^{(\mathbf{1 0})}$.

Yokoyama ad colleagues needed 3 years using functional end point (VF: MD slope) ${ }^{(11)}$. However, Quaranta and Floriani have found that (36.4\%) of their subjects assigned to brimonidine therapy have dropped out before completing the first year of their study in relation to subjects assigned timolol ( $10.1 \%)$ (12). The size of the population in this study can be affected if such a percentage $(36.4 \%)$ may be lost. Moreover, the effect age related decline on OCT results regarding RNFL ${ }^{(13)}$ and RGCC ${ }^{(14)}$. So, in this study, the follow up period was 6 months. +/- 2 weeks.

The first-line treatment of glaucoma usually begins with the use of a topical selective or nonselective blocker or a prostaglandin analog as a monotherapy. Second-line drugs of choice include alpha-agonists and topical carbonic anhydrase inhibitors ${ }^{(\mathbf{1 5})}$. However, it is accepted that there are scenarios when it may be more advisable to start with a fixed combination. Such as high baseline IOP or significant nerve damage that is not likely to reach target IOP on a single agent ${ }^{(\mathbf{1 6})}$. Moreover, according to the Ocular Hypertension Treatment Study (OHTS), Kass and colleagues have found that in $40 \%$ of patients with IOP ranging from 24 to $32 \mathrm{~mm} \mathrm{Hg}$, monotherapy (including beta blockers, prostaglandin analogues and topical carbonic anhydrase inhibitors) have failed to achieve $20 \%$ IOP reduction ${ }^{(17)}$.
Brimonidine may not achieve proper IOP control as a monotherapy to induce IOP reduction near $30 \%$ of baseline, so the only commercially available fixed combination at time of initiating this study was brimonidine/timolol $(0.2 \% / 0.5 \%)$ fixed combination. Moreover, the comparable IOP reduction $(29.9 \%$ for dorzolamide/timolol fixed combination and $28.1 \%$ for brimonidine/timolol fixed combination) ${ }^{(18)}$, can provide a comparable control group as timolol $0.5 \%$ is found in both groups helping to ensure uniformity of the treatment.

Neuroprotection for glaucoma refers to any intervention, independent of IOP reduction, which protects the optic nerve or prevents the death of retinal ganglion cells ${ }^{(\mathbf{1 6})}$. Thus, comparable (preferably equal) IOP reduction between the study and control groups is mandatory to reveal any pressure independent effect in the study group (Neuroprotection).

Krupin and colleagues have noticed a neuroprotective role of brimonidine in the LowPressure Glaucoma Treatment Study (LoGTS), when post treatment IOP was similar in both study and control groups ${ }^{\left({ }^{(19)}\right.}$. Janulevičiene and colleagues have found no neuroprotective effects according to visual field testing ${ }^{(20)}$. Lanza and colleagues have studied the neuroprotective effect of oral citicoline therapy in primary open-angle glaucoma (POAG). They found that IOP values showed no intra-group significant difference throughout the follow-up period in both groups ${ }^{(21)}$.

In this study, no significant difference between the study group and the control group regarding baseline mean intraocular pressure (24.79 and 25.06 $\mathrm{mm} \mathrm{Hg}$ respectively). The mean change in intraocular pressure in the two groups at different follow up visits was also matched without significant difference ( $p$ $>0.05$ ). Moreover, the overall change in intraocular pressure in the two groups at the end of the follow up showed insignificant difference $(p>0.05)$ as the net decrease in IOP in the study group and control group was -7.33 and $-7.52 \mathrm{mmHg}$ respectively from the base line. IOP decrease was $29.56 \%$ in the study group and $30 \%$ in the control group.

\section{Visual field testing:}

Krupin and colleagues have found that fewer brimonidine $0.2 \%$ treated patients $(9,9.1 \%)$ had visual field progression than timolol $0.5 \%$ treated patients $(31, \quad 39.2 \%)$ using PROGRESSOR software (sensitivity pointwise linear regression analysis) ${ }^{(19)}$.

Yokoyama and colleagues have found an improvement in MD slope from $-1.22 \mathrm{~dB} / \mathrm{y}$ to -0.38 $\mathrm{dB} / \mathrm{y}$ in comparison to timolol (from- $1.08 \mathrm{~dB} / \mathrm{y}$ to only $-0.52 \mathrm{~dB} / \mathrm{y}$ ) despite similar IOP reduction ${ }^{(11)}$.

In a study that included 33 eyes with open angle glaucoma Mohamed and Abo-Elkhie have found improvements in visual field MD values (from -7.2 to 3.3). PSD values have also been founded to be improved (from 4.6 to 3.4) after addition of brimonidine $0.2 \%$ eye drops twice daily o their treatment. This improvement was attributed to a 
neuroprotection action of brimonidine ${ }^{(22)}$. However, this improvement may not be purely attributed to neuroprotection as it was accompanied by IOP reduction (from 15.3 to $11.7 \mathrm{mmHg}$ ). While neuroprotection should be independent of IOP reduction ${ }^{(16,23)}$.

Moreover, the EMGT revealed that a 2-3 $\mathrm{mm}$ $\mathrm{Hg}$ decrease in IOP may decelerate the rate of MD progression by $30 \%{ }^{(24)}$.

To study the neuroprotective effect of oral citicoline therapy in primary open-angle glaucoma, Lanza and colleagues have used VF testing to reveal the neuroprotective role of citicoline. Without causing any change on IOP, citicoline had an effect in slowing the $\mathrm{MD}$ reduction (from baseline $-6.51 \mathrm{~dB}$ to -7.25 $\mathrm{dB})$ after 18 months of therapy and that this effect appears to be stable in the following 6 months. In the control group, MD reduction continued (from baseline -6.39 to $-9.28 \mathrm{~dB})^{(21)}$.

In this study, an insignificant difference ( $P>0.05)$ was found in the absolute change in MD between study and control groups $(-0.111 \mathrm{~dB}+/-1.04$ and $-0.246 \mathrm{~dB}+/-1.02$ respectively). Moreover, no statistically significant difference between MD changes between both groups has been found throughout the follow up visits.

Regarding VFI in this study, the absolute change showed an insignificant difference $(\mathrm{P}>0.05)$ between the study group $(-0.5882+/-2.07)$ and control group (-1.1471+/-0.061).

Regarding PSD in this study, the absolute change showed insignificant difference $(\mathrm{P}>0.05)$ between the study group $(0.173+/-0.759)$ and control group (0.1894+/-0.56).

The concept of a 'functional reserve' described a functional latency period in the natural history of glaucoma where structural change occurred without functional change ${ }^{(25)}$. Neighboring retinal ganglion cells compensate for cells nearby that are dead or dying until most of the cells in a region are not functional anymore, and only at this point is a change in functional vision detectable. SAP is not sensitive enough detect these very early glaucomatous changes due to this redundancy ${ }^{(26)}$. Moreover, redundancy in the sensory system may include the ability of the visual cortex to fill-in loss of visual field ${ }^{(27)}$. SAP may not be sensitive enough detect these very early glaucomatous changes due to this redundancy ${ }^{(26)}$.

VFI correlated linearly with MD in patients with moderate and advanced disease. However, this correlation was weaker in mild disease, as some patients with early disease had very high VFI values (ceiling effect). So, the diagnostic sensitivity may be diminished in early glaucoma ${ }^{(28)}$. Moreover, Gardiner and coworker showed the MD may detect progression more than1 year earlier than the VFI and more than 3 years earlier than the PSD ${ }^{(29)}$. Thus, in this study, VFI may not be sensitive to elaborate progression difference between study (MD -4.06) and control (MD -4.78) groups.
In one study, PSD has been used alongside with MD to study the neuroprotective role of ginkgo biloba intake for a mean of 2 years on NTG patients. Both global indices have given promising results. MD improved from -5.25 to $-4.31 \mathrm{~dB}$, while PSD improved from 4.71 to $4.48^{(30)}$.

OCT:

Circumpapillary RNFL has been the structural end point of choice in previous neuroprotection studies.

In their study, Tsai and Chang, have used the Scanning laser polarimetry Results revealed that the average RNFL did not change in a group of POAG patients after one year of twice daily brimonidine tartrate $0.2 \%$. The inferior segment underwent a decrease of $-1.4 \mathrm{um}$ while the superior segment a decrease of -1.1 um. compared to a decrease of -4.3 and -2.1 um respectively in POAG patients treated with timolol maleate $0.5 \%{ }^{(10)}$.

Lanza and colleagues have utilized RTVue OCT jointly with SAP to evaluate Citicoline neuroprotective effect in glaucoma, which was evident only after 18 months of therapy. Moreover citicoline was an adjunctive oral therapy to the topical hypotensive therapy ${ }^{(21)}$.

Rossetti and colleagues have studied the neuroprotective effect of citicoline eye drops using Spectralis OCT. Average RNFL measurements were assessed at baseline and every 6 months. Average RNFL thickness showed $-1.86 \mathrm{u}$ decrease in citicoline group after 3 years, versus $2.99 \mu \mathrm{m}$ in the placebo group. However, some potential confounders were found. The IOP-lowering regimen was modified in 25 patients $(32 \%)$ to control IOP in both groups. Modifications included laser trabeculoplasty, trabeculectomy, shifting from monotherapy to a fixed combination and from a fixed combination to a 3-drug regimen. Finally, their cohort was not POAG only, pseudoexfoliative glaucoma patients and surgically treated cases were also included ${ }^{(31)}$.

In this study, the neuroprotective effect of brimonidine was structurally evident within six months. Brimonidine was not used as an adjunctive therapy. It was rather used as an ocular hypotensive role with an additional neuroprotective effect.

The absolute change showed a significant $(\mathrm{P}<0.05)$ difference between the study and the control group as the study group showed lesser decrease in average RNFL while the control showed greater decrease in average RNFL, (-0.7273 um+/-2.2118 and -2.2121 um $+/-2.60717$ respectively).

The absolute change showed a significant difference $(\mathrm{P}<0.05)$ between the study and the control group as the study group showed lesser decrease in inferior RNFL while the control showed greater decrease in inferior RNFL, (-0.735um +/- 1.7975 and 1.559 um +/- 1.5214 respectively).

The absolute change showed a significant difference $(\mathrm{P}<0.05)$ between the study and the control group as the study group showed lesser decrease in 
superior RNFL while the control showed greater decrease in superior RNFL, (-0.6364um +/-1.36515 and -1.4848 um $+/-1.43878$ respectively).

The absolute change showed a significant difference $(\mathrm{P}<0.05)$ between the study and the control group as the study group showed lesser decrease in rim area while the control showed greater decrease in rim area , $(-0.0162 \mathrm{~mm} 2+/-0.07909$ and $-0.0797 \mathrm{~mm} 2+/-$ 0.15083 respectively).

The absolute change showed a significant $(\mathrm{P}>0.05)$ difference between study group and the control group as the study group showed a decrease in average $\mathrm{CD}$ ratio $(-0.01765+/-0.073405)$ while the control group showed an increase in average $\mathrm{CD}$ ratio and $0.01824+/-0.045625$ ).

The absolute change showed a significant $(\mathrm{P}<0.05)$ difference between study group and the control group as the study group showed a decrease in VCD ratio (-0.0167 +/-0.08859) while the control group showed an increase in VCD ratio $(0.0321+/-$ .09048).

The absolute change showed an insignificant $(\mathrm{P}>0.05)$ difference between the study and control group in Cup Volume $(0.00312 \mathrm{~mm} 3+/-0.061434$ and $0.00759 \mathrm{~mm} 3+/-0.066412$ increase respectively).

The absolute change showed an insignificant $(\mathrm{P}>0.05)$ difference between the study group and the control group as the study group showed a lesser decrease while the control group showed greater decrease in Average GCIPL thickness (-0.9412 um +/2.48561 and $-1.7059 \mathrm{um}+/-2.55283$ respectively).

The absolute change showed an insignificant $(\mathrm{P}>0.05)$ difference between the study group and the control group as the study group showed a lesser decrease while the control group showed greater decrease in Minimum GCIPL thickness (-0.781 um +/1.969 and $-1.271 \mathrm{um}+/-2.270$ respectively).

The main limitations of this study include small sample size and short follow up duration. The study was unmasked. The included cohort were within the mild glaucoma stage where structure - function relationship may not be clear ${ }^{(26)}$. Which was reflected in this study on the statistically insignificant results of SAP (24-2) and the statistically significant SD-OCT results. However, in accordance with this conclusion, Rossetti and colleagues have found a statistically significant evidence the neuroprotective effect of citicoline eye drops using HFA 10-2 progression rates, but not for 24-2 rates ${ }^{(31)}$.

Hood and colleagues have showed that macular damage occurs in about $90 \%$ of patients with early glaucoma, and macular damage within $10^{\circ}$ may be recognized as a common disease feature. Most of macular damage is arcuate in nature and often associated with local RNFL thinning corresponding to the inferior quadrant of the disc, a region that is particularly susceptible to glaucomatous damage ${ }^{(32)}$.

Cirafici and colleagues have found that the use of the 10-2 VF perimetry for central VF testing more accurately represents visual function in the macula. The use of 24-2 VF testing could therefore be insufficient to accurately characterize central VF deficits due to its limited spatial sampling. Indeed, the distance between testing locations in the 24-2 perimetry is $6^{\circ}$, whereas testing locations are $2^{\circ}$ apart in 10-2 perimetry. While $24-2$ perimetry is useful in detecting peripheral glaucomatous damage such as nasal step or arcuate scotomas, 10-2 perimetry is likely more sensitive for the detection of paracentral scotomas ${ }^{(33)}$.

The Octopus Tendency Oriented Perimetry (TOP) G1 and the Frequency Doubling Technology (FDT) were used to follow up the effect of 2-4 months of brimonidine treatment on glaucomatous eyes. TOP G1 strategy showed an increase in mean sensitivity, while FDT showed no significant differences ${ }^{(34)}$.

In OAG patients, electrophysiological recordings may provide selective information about the retinal function and the neural conduction along the visual pathways. Unlike the visual field assessments, they may of advantage as being completely objective, hence more unbiased examination of the function of the visual pathway. This objectiveness is because cognitive factors (like tiredness, stress and others) or patient's motor skills (especially for the elder patients) are absent ${ }^{(35)}$.

\section{CONCLUSION}

It could be concluded that neuroprotection is an additive glaucoma treatment strategy aiming to prevent, retard or reverse apoptosis-associated neuronal cell death resulting from glaucoma apart from that achieved by IOP lowering. This strategy was challenged to provide clear structural and functional results.

Preclinical studies have clarified numerous mechanisms for neuroprotection that brimonidine may exhibit. Moreover, many clinical trials on brimonidine as a neuroprotection agent have been conducted either within or outside the field of glaucoma.

In this study, the use of visual field-testing parameters (MD, VFI and PSD) have revealed that brimonidine had insignificantly halted glaucoma progression. On the other hand, some OCT parameters (average RNFL thickness, inferior RNFL thickness, superior RNFL thickness, rim area, average cup disc ratio and vertical cup disc ratio) gave promising neuroprotective results for brimonidine, while other OCT parameters ( cup volume, average GCLIPL thickness and minimum GCLIPL thickness) gave statistically insignificant neuroprotective results for brimonidine.

\section{REFERENCES}

1. Tham Y, Li X, Wong T et al. (2014): Global prevalence of glaucoma and projections of glaucoma burden through 2040: a systematic review and metaanalysis. Ophthalmology, 121(11): 2081-2090.

2. Schlamp C, Li Y, Dietz J et al. (2006): Progressive ganglion cell loss and optic nerve degeneration in 
$\mathrm{DBA} / 2 \mathrm{~J}$ mice is variable and asymmetric. BMC Neuroscience, 7(1): 1-14.

3. Nucci C, Martucci A, Giannini C et al. (2018): Neuroprotective agents in the management of glaucoma. Eye, 32(5): 938-945.

4. Bagli E, Kitsos G (2011): Neuroprotective Agents in Glaucoma. The mistery of glaucoma. Available: www. intechopen. com: InTech.

5. Burke J, Schwartz M (1996): Preclinical evaluation of brimonidine. Survey of ophthalmology, 41: 9-18.

6. Quigley H (2012): Clinical trials for glaucoma neuroprotection are not impossible. Current Opinion in Ophthalmology, 23(2): 144-154.

7. Boia R, Ruzafa N, Aires I et al. (2020): Neuroprotective strategies for retinal ganglion cell degeneration: current status and challenges ahead. International Journal of Molecular Sciences, 21(7): 2262-66.

8. Casson R, Chidlow G, Ebneter A et al. (2012): Translational neuroprotection research in glaucoma: a review of definitions and principles. Clinical \& Experimental Ophthalmology, 40(4): 350-357.

9. Artes P, Chauhan B (2005): Longitudinal changes in the visual field and optic disc in glaucoma. Progress in Retinal and Eye Research, 24(3): 333-354.

10. Tsai J, Chang H (2005): Comparison of the effects of brimonidine $0.2 \%$ and timolol $0.5 \%$ on retinal nerve fiber layer thickness in ocular hypertensive patients: a prospective, unmasked study. Journal of Ocular Pharmacology \& Therapeutics, 21(6): 475-482.

11. Yokoyama $Y$, Kawasaki $\mathrm{R}$, Takahashi $\mathrm{H}$ et al. (2019): Effects of brimonidine and timolol on the progression of visual field defects in open-angle glaucoma: a single-center randomized trial. Journal of Glaucoma, 28(7): 575-79.

12. Quaranta L, Floriani I (2011): The rate of progression and ocular perfusion pressure in the Low-pressure Glaucoma Treatment Study. American Journal of Ophthalmology, 152(5): 880-885.

13. Leung $\mathrm{C}, \mathrm{Yu} \mathrm{M}$, Weinreb $\mathrm{R}$ et al. (2012): Retinal nerve fiber layer imaging with spectral-domain optical coherence tomography: patterns of retinal nerve fiber layer progression. Ophthalmology, 119(9): 1858-1866.

14. Leung C, Ye C, Weinreb $R$ et al. (2013): Impact of age-related change of retinal nerve fiber layer and macular thicknesses on evaluation of glaucoma progression. Ophthalmology, 120(12): 2485-2492.

15. Gupta $\mathbf{N}$, Fong J, Ang $\mathrm{L}$ et al. (2008): Retinal tau pathology in human glaucomas. Canadian Journal of Ophthalmology, 43(1): 53-60.

16. Harasymowycz P, Birt C, Gooi P et al. (2016): Medical management of glaucoma in the 21 st century from a Canadian perspective. Journal of ophthalmology, 2016: 6509809.

17. Kass M, Gordon M, Gao F et al. (2010): Delaying treatment of ocular hypertension: the ocular hypertension treatment study. Archives of Ophthalmology, 128(3): 276-81.

18. Cheng J, Cheng S, Gao L et al. (2012): Intraocular pressure-lowering effects of commonly used fixedcombination drugs with timolol: a systematic review and meta-analysis. PLoS One, 7(9): 45079.

19. Krupin T, Liebmann J, Greenfield D et al. (2011): A randomized trial of brimonidine versus timolol in preserving visual function: results from the Low-
Pressure Glaucoma Treatment Study. American Journal of Ophthalmology, 151(4): 671-681.

20. Janulevičiene I, Ehrlich R, Siesky B et al. (2011): Evaluation of hemodynamic parameters as predictors of glaucoma progression. Journal of Ophthalmology, 2011: 164320.

21. Lanza M, Gironi Carnevale U, Mele L et al. (2019): Morphological and functional evaluation of oral citicoline therapy in chronic open-angle glaucoma patients: A pilot study with a 2-year follow-up. Frontiers in Pharmacology, 10: 1117-1122.

22. Mohamed J, Abo-Elkhei O (2017): The Role of Brimonidine Eye Drops as an Adjunctive Therapy for Optic Nerve Protection in Patients with Controlled Open Angle Glaucoma. The Egyptian Journal of Hospital Medicine, 68(3): 1418-1424.

23. Sena D, Lindsley K (2017): Neuroprotection for treatment of glaucoma in adults. Cochrane Database of Systematic Reviews, (1): 35-40.

24. Heijl A, Bengtsson B, Hyman L et al. (2009): Natural history of open-angle glaucoma. Ophthalmology, 116(12): 2271-2276.

25. Johnson C (1994): Selective versus nonselective losses in glaucoma. Journal of Glaucoma, 3: 32-44.

26. Lucy K, Wollstein G (2016): Structural and functional evaluations for the early detection of glaucoma. Expert Review of Ophthalmology, 11(5): 367-376.

27. De Moraes C, Liebmann J, Levin L (2017): Detection and measurement of clinically meaningful visual field progression in clinical trials for glaucoma. Progress in Retinal and Eye Research, 56: 107-147.

28. Sousa M, Biteli L, Dorairaj S et al. (2015): Suitability of the Visual Field Index according to glaucoma severity. Journal of Current Glaucoma Practice, 9(3): 65-71.

29. Gardiner S, Demirel S (2017): Detecting change using standard global perimetric indices in glaucoma. American Journal of Ophthalmology, 176: 148-156.

30. Shim S, Xia C, Zhong G et al. (2012): Superresolution fluorescence imaging of organelles in live cells with photoswitchable membrane probes. Proceedings of the National Academy of Sciences, 109(35): 13978-13983.

31. Rossetti L, Iester M, Tranchina $L$ et al. (2020): Can treatment with Citicoline Eyedrops reduce progression in glaucoma? The results of a randomized placebocontrolled clinical trial. Journal of Glaucoma, 29(7): 513-517.

32. Hood D, Raza A, de Moraes C et al. (2013): Glaucomatous damage of the macula. Progress in Retinal and Eye Research, 32: 1-21.

33. Cirafici P, Maiello G, Ancona C et al. (2021): Pointwise correlations between 10-2 Humphrey visual field and OCT data in open angle glaucoma. Eye, 35(3): 868876.

34. Lafuente M, Villegas-Pérez M, Sobrado-Calvo $P$ et al. (2001): Neuroprotective effects of $\alpha 2$-selective adrenergic agonists against ischemia-induced retinal ganglion cell death. Investigative Ophthalmology \& Visual Science, 42(9): 2074-2084.

35. Firan A, Istrate $S$, Iancu $R$ et al. (2020): Visual evoked potential in the early diagnosis of glaucoma. Literature review. Romanian Journal of Ophthalmology, 64(1): 15-22. 